\title{
CONVERGENCE THEOREMS FOR SET-VALUED DENJOY-PETTIS INTEGRABLE MAPPINGS
}

\author{
Chun-Kee Park
}

\begin{abstract}
In this paper, we introduce the Denjoy-Pettis integral of set-valued mappings and investigate some properties of the set-valued Denjoy-Pettis integral. Finally we obtain the Dominated Convergence Theorem and Monotone Convergence Theorem for set-valued DenjoyPettis integrable mappings.
\end{abstract}

\section{Introduction}

Saks [11] introduced the Denjoy integral of real-valued functions which is a natural extension of the Lebesgue integral. Gordon [8] introduced the DenjoyPettis integral of Banach-valued functions in terms of the Denjoy integral which is the Denjoy extension of Pettis integral. The notion of integral of set-valued mappings is very useful in many branches of mathematics like mathematical economics, control theory, convex analysis, etc. It has been introduced by many authors and in different ways. Several types of integrals of set-valued mappings were introduced and studied by Aumann [1], Cascales and Rodriguez [2], Debreu [3], Di Piazza and Musial [4, 5], El Amri and Hess [6], Papageoriou [10] and others.

In this paper, we introduce the Denjoy-Pettis integral of set-valued mappings and investigate some properties of the set-valued Denjoy-Pettis integral. Finally we obtain two convergence theorems for set-valued Denjoy-Pettis integrable mappings.

\section{Preliminaries}

Throughout this paper, $\mathcal{L}$ denotes the family of all Lebesgue measurable subsets of $[a, b]$ and $X$ a Banach space with dual $X^{*}$. The closed unit ball of $X^{*}$ is denoted by $B_{X^{*}} . C L(X)$ denotes the family of all nonempty closed subsets of $X, C(X)$ the family of all nonempty closed convex subsets of $X, C B(X)$ the family of all nonempty closed bounded convex subsets of $X, C W K(X)$ the family of all nonempty convex weakly compact subsets of $X$ and $C K(X)$

Received July 21, 2008.

2000 Mathematics Subject Classification. 26A39, 28B05, 28B20.

Key words and phrases. set-valued mapping, Denjoy integral, Denjoy-Pettis integral. 
the family of all nonempty convex compact subsets of $X$. Note that if $X$ is reflexive, then $C W K(X)=C B(X)$.

For $A \subseteq X$ and $x^{*} \in X^{*}$, let $s\left(x^{*}, A\right)=\sup \left\{x^{*}(x): x \in A\right\}$, the support function of $A$.

For $A, B \in C L(X)$, let $H(A, B)$ denote the Hausdorff metric of $A$ and $B$ defined by

$$
H(A, B)=\max \left(\sup _{a \in A} d(a, B), \sup _{b \in B} d(b, A)\right),
$$

where $d(a, B)=\inf _{b \in B}\|a-b\|$ and $d(b, A)=\inf _{a \in A}\|a-b\|$. Especially,

$$
H(A, B)=\sup _{\left\|x^{*}\right\| \leq 1}\left|s\left(x^{*}, A\right)-s\left(x^{*}, B\right)\right|
$$

whenever $A, B$ are convex sets. The number $\|A\|$ is defined by $\|A\|=H(A$, $\{0\})=\sup _{x \in A}\|x\|$. If $A \in C B(X)$ and $x_{1}^{*}, x_{2}^{*} \in X^{*}$, then

$$
\left|s\left(x_{1}^{*}, A\right)-s\left(x_{2}^{*}, A\right)\right| \leq\left\|x_{1}^{*}-x_{2}^{*}\right\|\|A\| .
$$

Note that $(C W K(X), H)$ is a complete metric space.

The mapping $F:[a, b] \rightarrow C L(X)$ is called a set-valued mapping. $F$ is said to be scalarly measurable if for every $x^{*} \in X^{*}$, the real-valued function $s\left(x^{*}, F(\cdot)\right)$ is measurable. $F$ is said to be measurable if $F^{-1}(A)=\{t \in[a, b]: F(t) \cap A \neq$ $\phi\} \in \mathcal{L}$ for every $A \in C L(X)$.

Let $F:[a, b] \rightarrow C L(X)$. Then the following statements are equivalent [10]:

(1) $F:[a, b] \rightarrow C L(X)$ is measurable;

(2) $F^{-1}(U)=\{t \in[a, b]: F(t) \cap U \neq \phi\} \in \mathcal{L}$ for every open subset $U$ of $X$;

(3) (Castaing representation) there exists a sequence $\left\{f_{n}\right\}$ of measurable functions $f_{n}:[a, b] \rightarrow X$ such that $F(t)=c l\left\{f_{n}(t)\right\}$ for all $t \in[a, b]$.

If $F:[a, b] \rightarrow C L(X)$ is measurable then $F:[a, b] \rightarrow C L(X)$ is scalarly measurable. Let $X$ be a separable Banach space. Then $F:[a, b] \rightarrow C W K(X)$ is measurable if and only if $F:[a, b] \rightarrow C W K(X)$ is scalarly measurable [6].

$f:[a, b] \rightarrow X$ is called a selection of $F:[a, b] \rightarrow C L(X)$ if $f(t) \in F(t)$ for all $t \in[a, b]$.

Definition 2.1 ([8]). Let $F:[a, b] \rightarrow X$ and let $E \subseteq[a, b]$.

(a) The function $F$ is said to be absolutely continuous on $E$ ( $F$ is AC on $E)$ if for each $\epsilon>0$ there exists $\delta>0$ such that $\sum_{i=1}^{n}\left\|F\left(d_{i}\right)-F\left(c_{i}\right)\right\|<\epsilon$ whenever $\left\{\left[c_{i}, d_{i}\right]: 1 \leq i \leq n\right\}$ is a finite collection of non-overlapping intervals that have endpoints in $E$ and satisfy $\sum_{i=1}^{n}\left(d_{i}-c_{i}\right)<\delta$.

(b) The function $F$ is said to be generalized absolutely continuous on $E$ ( $F$ is ACG on $E$ ) if $\mathrm{F}$ is continuous on $E$ and $E$ can be expressed as a countable union of sets on each of which $F$ is AC.

Definition 2.2 ([8]). Let $F:[a, b] \rightarrow X$ and let $t \in(a, b)$. A vector $z$ in $X$ is called the approximate derivative of $F$ at $t$ if there exists a measurable set $E \subseteq[a, b]$ that has $t$ as a point of density such that $\lim _{\substack{s \rightarrow t \\ s \in E}} \frac{F(s)-F(t)}{s-t}=z$. We will write $F_{a p}^{\prime}(t)=z$. 
A function $f:[a, b] \rightarrow \mathbb{R}$ is said to be Denjoy integrable on $[a, b]$ if there exists an ACG function $F:[a, b] \rightarrow \mathbb{R}$ such that $F_{a p}^{\prime}=f$ almost everywhere on $[a, b]$. In this case, we write $\int_{a}^{b} f(t) d t=F(b)-F(a)$.

The function $f$ is Denjoy integrable on a set $A \subseteq[a, b]$ if $f \chi_{A}$ is Denjoy integrable on $[a, b]$. In this case, we write $\int_{A} f(t) d t=\int_{a}^{b} f \chi_{A}(t) d t$.

Definition 2.3 ([8]). A function $f:[a, b] \rightarrow X$ is said to be Denjoy-Pettis integrable on $[a, b]$ if for each $x^{*} \in X^{*}$ the function $x^{*} f$ is Denjoy integrable on $[a, b]$ and if for every subinterval $[c, d]$ of $[a, b]$ there exists a vector $x_{[c, d]} \in X$ such that $x^{*}\left(x_{[c, d]}\right)=\int_{c}^{d} x^{*} f(t) d t$ for all $x^{*} \in X^{*}$. In this case, the vector $x_{[a, b]}$ is called the Denjoy-Pettis integral of $f$ on $[a, b]$ and is denoted by $(D P) \int_{a}^{b} f(t) d t$.

The function $f:[a, b] \rightarrow X$ is Denjoy-Pettis integrable on a set $A \subseteq[a, b]$ if the function $f \chi_{A}$ is Denjoy-Pettis integrable on $[a, b]$. In this case, we write $(D P) \int_{A} f(t) d t=(D P) \int_{a}^{b} f \chi_{A}(t) d t$.

Theorem $2.4([13])$. Let $A \in C(X)$. Then the support function $s(\cdot, A)$

(1) is positively homogeneous, i.e., $s\left(\lambda x^{*}, A\right)=\lambda s\left(x^{*}, A\right)$ for all $\lambda \geq 0$ and $x^{*} \in X^{*}$

(2) is a convex function on $X^{*}$;

(3) is weak* lower semi-continuous on $X^{*}$.

Conversely, if a function $\varphi: X^{*} \rightarrow[-\infty,+\infty]$ satisfies the conditions (1)(3), then there exists an $A \in C(X)$ such that $\varphi\left(x^{*}\right)=s\left(x^{*}, A\right)$ for each $x^{*} \in$ $X^{*}$. The set $A$ is unique and given by $A=\left\{x \in X: x^{*}(x) \leq \varphi\left(x^{*}\right)\right.$ for all $x^{*} \in$ $\left.X^{*}\right\}$.

Theorem 2.5 ([13]). If $A_{n} \in C W K(X)$ for each $n \in \mathbb{N}$ and $\lim _{n \rightarrow \infty} s\left(x^{*}, A_{n}\right)$ exists for each $x^{*} \in X^{*}$, then there exists an $M>0$ such that $\sup _{n \in \mathbb{N}}\left\|A_{n}\right\| \leq$ $M$.

\section{Results}

In this section, we introduce the Denjoy-Pettis integral of set-valued mappings and obtain some properties of the Denjoy-Pettis integral.

Definition 3.1. A set-valued mapping $F:[a, b] \rightarrow C W K(X)$ is said to be Denjoy-Pettis integrable on $[a, b]$ if for each $x^{*} \in X^{*} s\left(x^{*}, F(\cdot)\right)$ is Denjoy integrable on $[a, b]$ and for every subinterval $[c, d]$ of $[a, b]$ there exists $W_{[c, d]} \in$ $C W K(X)$ such that

$$
s\left(x^{*}, W_{[c, d]}\right)=\int_{c}^{d} s\left(x^{*}, F(t)\right) d t
$$

for each $x^{*} \in X^{*}$. We write $W_{[c, d]}=(D P) \int_{c}^{d} F(t) d t$. 
Note that when a set-valued mapping is a function $f:[a, b] \rightarrow X$, then the set $W_{[c, d]}$ is reduced to vector in $X$ and the equality (1) turns into

$$
x^{*}\left(W_{[c, d]}\right)=\int_{c}^{d} x^{*} f(t) d t
$$

for each $x^{*} \in X^{*}$. In this case, the function $f:[a, b] \rightarrow X$ is Denjoy-Pettis integrable on $[a, b]$.

The following two theorems follow easily from the properties of the Denjoy integral and the support function.

Theorem 3.2. Let $F:[a, b] \rightarrow C W K(X)$ and $c \in(a, b)$. Then

(1) if $F$ is Denjoy-Pettis integrable on $[a, b]$, then $F$ is Denjoy-Pettis integrable on every subinterval of $[a, b]$;

(2) if $F$ is Denjoy-Pettis integrable on $[a, c]$ and $[c, b]$, then $F$ is DenjoyPettis integrable on $[a, b]$ and

$$
(D P) \int_{a}^{b} F(t) d t=(D P) \int_{a}^{c} F(t) d t+(D P) \int_{c}^{b} F(t) d t .
$$

Theorem 3.3. Let $F:[a, b] \rightarrow C W K(X)$ and $G:[a, b] \rightarrow C W K(X)$ be Denjoy-Pettis integrable on $[a, b]$ and $\lambda \geq 0$. Then

(1) $F+G$ is Denjoy-Pettis integrable on $[a, b]$ and

$$
(D P) \int_{a}^{b}\{F(t)+G(t)\} d t=(D P) \int_{a}^{b} F(t) d t+(D P) \int_{a}^{b} G(t) d t
$$

(2) $\lambda F$ is Denjoy-Pettis integrable on $[a, b]$ and

$$
(D P) \int_{a}^{b} \lambda F(t) d t=\lambda(D P) \int_{a}^{b} F(t) d t
$$

Theorem 3.4. Let $f:[a, b] \rightarrow X$ be Denjoy-Pettis integrable on $[a, b]$ and $F:[a, b] \rightarrow C W K(X)$ and $G:[a, b] \rightarrow C W K(X)$ be Denjoy-Pettis integrable on $[a, b]$. Then

(1) if $f(t) \in F(t)$ a.e., then

$$
(D P) \int_{a}^{b} f(t) d t \in(D P) \int_{a}^{b} F(t) d t
$$

(2) if $F(t) \subseteq G(t)$ a.e., then $(D P) \int_{a}^{b} F(t) d t \subseteq(D P) \int_{a}^{b} G(t) d t$;

(3) if $F(t)=G(t)$ a.e., then $(D P) \int_{a}^{b} F(t) d t=(D P) \int_{a}^{b} G(t) d t$.

Proof. (1) Since $f:[a, b] \rightarrow X$ is Denjoy-Pettis integrable on $[a, b]$, for each $x^{*} \in X^{*} x^{*} f$ is Denjoy integrable on $[a, b]$ and

$$
\int_{a}^{b} x^{*} f(t) d t=x^{*}\left((D P) \int_{a}^{b} f(t) d t\right)
$$


If $f(t) \in F(t)$ a.e., then for each $x^{*} \in X^{*}$

$$
\begin{aligned}
x^{*}\left((D P) \int_{a}^{b} f(t) d t\right) & =\int_{a}^{b} x^{*} f(t) d t \\
& \leq \int_{a}^{b} s\left(x^{*}, F(t)\right) d t \\
& =s\left(x^{*},(D P) \int_{a}^{b} F(t) d t\right) .
\end{aligned}
$$

Since $(D P) \int_{a}^{b} F(t) d t \in C W K(X)$, by the separation theorem

$$
(D P) \int_{a}^{b} f(t) d t \in(D P) \int_{a}^{b} F(t) d t
$$

(2) If $F(t) \subseteq G(t)$ a.e., then for each $x^{*} \in X^{*}$

$$
\int_{a}^{b} s\left(x^{*}, F(t)\right) d t \leq \int_{a}^{b} s\left(x^{*}, G(t)\right) d t
$$

and so

$$
s\left(x^{*},(D P) \int_{a}^{b} F(t) d t\right) \leq s\left(x^{*},(D P) \int_{a}^{b} G(t) d t\right) .
$$

Since $(D P) \int_{a}^{b} F(t) d t,(D P) \int_{a}^{b} G(t) d t \in C W K(X)$, by the separation theorem

$$
(D P) \int_{a}^{b} F(t) d t \subseteq(D P) \int_{a}^{b} G(t) d t .
$$

(3) The proof is similar to (1).

Theorem 3.5. Let $X$ be a separable Banach space. If $F:[a, b] \rightarrow C W K(X)$ is Denjoy-Pettis integrable on $[a, b]$, then $F:[a, b] \rightarrow C W K(X)$ is measurable on $[a, b]$.

Proof. If $F:[a, b] \rightarrow C W K(X)$ is Denjoy-Pettis integrable on $[a, b]$, then $s\left(x^{*}, F(\cdot)\right)$ is Denjoy integrable on $[a, b]$ and so $s\left(x^{*}, F(\cdot)\right)$ is measurable on $[a, b]$ for each $x^{*} \in X^{*}$ by $[9$, Theorem 15.8]. Thus $F:[a, b] \rightarrow C W K(X)$ is scalarly measurable on $[a, b]$. Hence $F:[a, b] \rightarrow C W K(X)$ is measurable on $[a, b]$.

$F:[a, b] \rightarrow C L(X)$ is said to be Denjoy integrably bounded on $[a, b]$ if there exists a Denjoy integrable real-valued function $h$ on $[a, b]$ such that for each $t \in[a, b],\|x\| \leq h(t)$ for all $x \in F(t)$.

Theorem 3.6. Let $X$ be a separable Banach space. If $F:[a, b] \rightarrow C W K(X)$ and $G:[a, b] \rightarrow C W K(X)$ are Denjoy integrably bounded and Denjoy-Pettis integrable on $[a, b]$, then $H(F, G)$ is Denjoy integrable on $[a, b]$ and

$$
H\left((D P) \int_{a}^{b} F(t) d t,(D P) \int_{a}^{b} G(t) d t\right) \leq \int_{a}^{b} H(F(t), G(t)) d t
$$


Proof. Since $F$ and $G$ are measurable, there exist Castaing representations $\left\{f_{n}\right\}$ and $\left\{g_{n}\right\}$ for $F$ and $G$. Since $f_{n}$ and $g_{n}$ are measurable for all $n \in \mathbb{N}$,

$$
H(F(t), G(t))=\max \left(\sup _{n \geq 1} \inf _{k \geq 1}\left\|f_{n}(t)-g_{k}(t)\right\|, \sup _{n \geq 1} \inf _{k \geq 1}\left\|g_{n}(t)-f_{k}(t)\right\|\right)
$$

is measurable. Since $F$ and $G$ are Denjoy integrably bounded on $[a, b]$, there exist Denjoy integrable real-valued functions $h_{1}$ and $h_{2}$ such that for each $t \in[a, b],\|x\| \leq h_{1}(t)$ for all $x \in F(t)$ and $\|x\| \leq h_{2}(t)$ for all $x \in G(t)$. Since $h_{1}$ and $h_{2}$ are nonnegative and Denjoy integrable on $[a, b], h_{1}$ and $h_{2}$ are Lebesgue integrable on $[a, b]$. Hence we have

$$
H(F(t), G(t)) \leq H(F(t),\{0\})+H(G(t),\{0\}) \leq h_{1}(t)+h_{2}(t)
$$

for each $t \in[a, b]$. Therefore $H(F, G)$ is Lebesgue integrable on $[a, b]$ and so $H(F, G)$ is Denjoy integrable on $[a, b]$. Hence we have

$$
\begin{aligned}
& H\left((D P) \int_{a}^{b} F(t) d t,(D P) \int_{a}^{b} G(t) d t\right) \\
= & \sup _{\left\|x^{*}\right\| \leq 1}\left|s\left(x^{*},(D P) \int_{a}^{b} F(t) d t\right)-s\left(x^{*},(D P) \int_{a}^{b} G(t) d t\right)\right| \\
= & \sup _{\left\|x^{*}\right\| \leq 1}\left|\int_{a}^{b} s\left(x^{*}, F(t)\right) d t-\int_{a}^{b} s\left(x^{*}, G(t)\right) d t\right| \\
\leq & \sup _{\left\|x^{*}\right\| \leq 1} \int_{a}^{b}\left|s\left(x^{*}, F(t)\right)-s\left(x^{*}, G(t)\right)\right| d t \\
\leq & \int_{a}^{b} \sup _{\left\|x^{*}\right\| \leq 1}\left|s\left(x^{*}, F(t)\right)-s\left(x^{*}, G(t)\right)\right| d t \\
= & \int_{a}^{b} H(F(t), G(t)) d t .
\end{aligned}
$$

Theorem 3.7. Let $X$ be a reflexive and separable Banach space and let $F_{n}$ : $[a, b] \rightarrow C W K(X)$ be a Denjoy-Pettis integrable set-valued mapping for each $n \in \mathbb{N}$ and let $F:[a, b] \rightarrow C W K(X)$ be a set-valued mapping such that $\lim _{n \rightarrow \infty} H\left(F_{n}(t), F(t)\right)=0$ on $[a, b]$. If there exists a Denjoy integrable function $h$ on $[a, b]$ such that $\left\|F_{n}(t)\right\| \leq h(t)$ on $[a, b]$ for each $n \in \mathbb{N}$, then $F:[a, b] \rightarrow C W K(X)$ is Denjoy-Pettis integrable on $[a, b]$ and

$$
\lim _{n \rightarrow \infty} H\left((D P) \int_{a}^{b} F_{n}(t) d t,(D P) \int_{a}^{b} F(t) d t\right)=0 .
$$

Proof. Since $F_{n}:[a, b] \rightarrow C W K(X)$ is Denjoy-Pettis integrable on $[a, b]$ for each $n \in \mathbb{N}$, for each $n \in \mathbb{N}, s\left(x^{*}, F_{n}(\cdot)\right)$ is Denjoy integrable on $[a, b]$ for each 
$x^{*} \in X^{*}$ and for every subinterval $[c, d]$ of $[a, b]$ there exists $(D P) \int_{c}^{d} F_{n}(t) d t \in$ $C W K(X)$ such that

$$
s\left(x^{*},(D P) \int_{c}^{d} F_{n}(t) d t\right)=\int_{c}^{d} s\left(x^{*}, F_{n}(t)\right) d t
$$

for each $x^{*} \in X^{*}$. Since $\lim _{n \rightarrow \infty} H\left(F_{n}(t), F(t)\right)=\lim _{n \rightarrow \infty} \sup _{\left\|x^{*}\right\| \leq 1} \mid s\left(x^{*}\right.$, $\left.F_{n}(t)\right)-s\left(x^{*}, F(t)\right) \mid=0$ on $[a, b], \lim _{n \rightarrow \infty}\left|s\left(x^{*}, F_{n}(t)\right)-s\left(x^{*}, F(t)\right)\right|=0$ on $[a, b]$ for each $x^{*} \in B_{X^{*}}$ and so $x^{*} \in X^{*}$. Thus $\lim _{n \rightarrow \infty} s\left(x^{*}, F_{n}(t)\right)=$ $s\left(x^{*}, F(t)\right)$ on $[a, b]$ for each $x^{*} \in X^{*}$. For each $n \in \mathbb{N},\left|s\left(x^{*}, F_{n}(t)\right)\right| \leq$ $\sup _{\left\|x^{*}\right\| \leq 1}\left|s\left(x^{*}, F_{n}(t)\right)\right|=H\left(F_{n}(t),\{0\}\right)=\left\|F_{n}(t)\right\| \leq h(t)$ on $[a, b]$ for each $x^{*} \in B_{X^{*}}$. Hence $\left|s\left(x^{*}, F_{n}(t)\right)\right| \leq\left\|x^{*}\right\| h(t)$ on $[a, b]$ for each $x^{*} \in X^{*}$. Since $h$ is Denjoy integrable on $[a, b],\left\|x^{*}\right\| h$ is Denjoy integrable on $[a, b]$ for each $x^{*} \in X^{*}$. By the Dominated Convergence Theorem for the Denjoy integral, for each $x^{*} \in X^{*}, s\left(x^{*}, F(\cdot)\right)$ is Denjoy integrable on $[a, b]$ and for every subinterval $[c, d]$ of $[a, b]$

$$
\lim _{n \rightarrow \infty} \int_{c}^{d} s\left(x^{*}, F_{n}(t)\right) d t=\int_{c}^{d} s\left(x^{*}, F(t)\right) d t .
$$

Let $[c, d]$ be any subinterval of $[a, b]$. We define a function $\varphi: X^{*} \rightarrow \mathbb{R}$ by $\varphi\left(x^{*}\right)=\int_{c}^{d} s\left(x^{*}, F(t)\right) d t$. Then $\varphi$ is positively homogeneous and convex. Now we prove that $\varphi$ is continuous on $X^{*}$. $(D P) \int_{c}^{d} F_{n}(t) d t \in C W K(X)$ for each $n \in \mathbb{N}$ and $\lim _{n \rightarrow \infty} s\left(x^{*},(D P) \int_{c}^{d} F_{n}(t) d t\right)=\lim _{n \rightarrow \infty} \int_{c}^{d} s\left(x^{*}, F_{n}(t)\right) d t=$ $\int_{c}^{d} s\left(x^{*}, F(t)\right) d t$ exists for each $x^{*} \in X^{*}$. By Theorem 2.5 there exists an $M>0$ such that $\sup _{n \in \mathbb{N}}\left\|(D P) \int_{c}^{d} F_{n}(t) d t\right\| \leq M$. For each $\epsilon>0$ let $\delta=\epsilon / M$. If $x_{1}^{*}, x_{2}^{*} \in X^{*},\left\|x_{1}^{*}-x_{2}^{*}\right\|<\delta$, then

$$
\begin{aligned}
\left|\varphi\left(x_{1}^{*}\right)-\varphi\left(x_{2}^{*}\right)\right| & =\left|\int_{c}^{d} s\left(x_{1}^{*}, F(t)\right) d t-\int_{c}^{d} s\left(x_{2}^{*}, F(t)\right) d t\right| \\
& =\lim _{n \rightarrow \infty}\left|\int_{c}^{d} s\left(x_{1}^{*}, F_{n}(t)\right) d t-\int_{c}^{d} s\left(x_{2}^{*}, F_{n}(t)\right) d t\right| \\
& =\lim _{n \rightarrow \infty}\left|s\left(x_{1}^{*},(D P) \int_{c}^{d} F_{n}(t) d t\right)-s\left(x_{2}^{*},(D P) \int_{c}^{d} F_{n}(t) d t\right)\right| \\
& \leq \lim _{n \rightarrow \infty}\left\|x_{1}^{*}-x_{2}^{*}\right\| \|(D P) \int_{c}^{d} F_{n}(t) d t|| \\
& \leq M\left\|x_{1}^{*}-x_{2}^{*}\right\| \\
& <M \delta=\epsilon .
\end{aligned}
$$

Thus $\varphi$ is continuous on $X^{*}$. By Theorem 2.4 there exists $W_{[c, d]} \in C(X)$ such that $\varphi\left(x^{*}\right)=s\left(x^{*}, W_{[c, d]}\right)$ for each $x^{*} \in X^{*}$. Since $\left|\varphi\left(x^{*}\right)\right|=\left|\int_{c}^{d} s\left(x^{*}, F(t)\right) d t\right|$ $<\infty$ for each $x^{*} \in X^{*}, W_{[c, d]} \in C B(X)$ by the Resonance Theorem. Since 
$X$ is reflexive, $W_{[c, d]} \in C W K(X)$ and $s\left(x^{*}, W_{[c, d]}\right)=\varphi\left(x^{*}\right)=\int_{c}^{d} s\left(x^{*}, F(t)\right) d t$ for each $x^{*} \in X^{*}$. Hence $F:[a, b] \rightarrow C W K(X)$ is Denjoy-Pettis integrable on $[a, b]$ and $W_{[c, d]}=(D P) \int_{c}^{d} F(t) d t$.

Since $\lim _{n \rightarrow \infty} H\left(F_{n}(t), F(t)\right)=0$ on $[a, b]$, for each $\epsilon>0$ and $t \in[a, b]$ there exists $N \in \mathbb{N}$ such that $n \geq N \Rightarrow H\left(F_{n}(t), F(t)\right)<\epsilon$. For some $n \in \mathbb{N}$ with $n \geq N$,

$$
\begin{aligned}
\|F(t)\| & =H(F(t),\{0\}) \leq H\left(F(t), F_{n}(t)\right)+H\left(F_{n}(t),\{0\}\right) \\
& <\left\|F_{n}(t)\right\|+\epsilon \leq h(t)+\epsilon .
\end{aligned}
$$

Since $\epsilon>0$ is arbitrary, $\|F(t)\| \leq h(t)$ on $[a, b]$, i.e., $F:[a, b] \rightarrow C W K(X)$ is Denjoy integrably bounded on $[a, b]$. By Theorem 3.6 $H\left(F_{n}, F\right)$ is Denjoy integrable on $[a, b]$ and

$$
H\left((D P) \int_{a}^{b} F_{n}(t) d t,(D P) \int_{a}^{b} F(t) d t\right) \leq \int_{a}^{b} H\left(F_{n}(t), F(t)\right) d t
$$

for each $n \in \mathbb{N}$. By the Dominated Convergence Theorem for the Denjoy integral we have

$\lim _{n \rightarrow \infty} H\left((D P) \int_{a}^{b} F_{n}(t) d t,(D P) \int_{a}^{b} F(t) d t\right) \leq \lim _{n \rightarrow \infty} \int_{a}^{b} H\left(F_{n}(t), F(t)\right) d t=0$.

Thus

$$
\lim _{n \rightarrow \infty} H\left((D P) \int_{a}^{b} F_{n}(t) d t,(D P) \int_{a}^{b} F(t) d t\right)=0 .
$$

Let $F_{n}:[a, b] \rightarrow C W K(X)$ be a set-valued mapping for each $n \in \mathbb{N}$. The sequence $\left\{F_{n}\right\}$ is said to be monotone increasing (resp. monotone decreasing) if for each $n \in \mathbb{N} F_{n}(t) \subseteq F_{n+1}(t)$ (resp. $\left.F_{n}(t) \supseteq F_{n+1}(t)\right)$ for all $t \in[a, b]$. The sequence $\left\{F_{n}\right\}$ is said to be monotone if it is monotone increasing or monotone decreasing.

Theorem 3.8. Let $X$ be a reflexive and separable Banach space and let $\left\{F_{n}\right\}$ be a monotone sequence of Denjoy-Pettis integrable $C W K(X)$-valued mappings on $[a, b]$ and let $F:[a, b] \rightarrow C W K(X)$ be a set-valued mapping such that $H\left(F_{1}, F\right)$ is bounded and $\lim _{n \rightarrow \infty} H\left(F_{n}(t), F(t)\right)=0$ on $[a, b]$. If $\lim _{n \rightarrow \infty}(D P) \int_{a}^{b} F_{n}(t) d t$ $\in C W K(X)$, then $F:[a, b] \rightarrow C W K(X)$ is Denjoy-Pettis integrable on $[a, b]$ and

$$
\lim _{n \rightarrow \infty} H\left((D P) \int_{a}^{b} F_{n}(t) d t,(D P) \int_{a}^{b} F(t) d t\right)=0 .
$$

Proof. Assume that $\left\{F_{n}\right\}$ is a monotone increasing sequence of Denjoy-Pettis integrable $C W K(X)$-valued mappings on $[a, b]$. The proof is similar when $\left\{F_{n}\right\}$ is a monotone decreasing sequence of Denjoy-Pettis integrable $C W K(X)$ valued mappings on $[a, b]$. Since $F_{n}:[a, b] \rightarrow C W K(X)$ is Denjoy-Pettis integrable on $[a, b]$ for each $n \in \mathbb{N}$, for each $n \in \mathbb{N}, s\left(x^{*}, F_{n}(\cdot)\right)$ is Denjoy 
integrable on $[a, b]$ for each $x^{*} \in X^{*}$ and for every subinterval $[c, d]$ of $[a, b]$ there exists $(D P) \int_{c}^{d} F_{n}(t) d t \in C W K(X)$ such that

$$
s\left(x^{*},(D P) \int_{c}^{d} F_{n}(t) d t\right)=\int_{c}^{d} s\left(x^{*}, F_{n}(t)\right) d t
$$

for each $x^{*} \in X^{*}$. Since $\lim _{n \rightarrow \infty} H\left(F_{n}(t), F(t)\right)=\lim _{n \rightarrow \infty} \sup _{\left\|x^{*}\right\| \leq 1} \mid s\left(x^{*}\right.$, $\left.F_{n}(t)\right)-s\left(x^{*}, F(t)\right) \mid=0$ on $[a, b], \lim _{n \rightarrow \infty}\left|s\left(x^{*}, F_{n}(t)\right)-s\left(x^{*}, F(t)\right)\right|=0$ on $[a, b]$ for each $x^{*} \in B_{X^{*}}$ and so $x^{*} \in X^{*}$. Thus $\lim _{n \rightarrow \infty} s\left(x^{*}, F_{n}(t)\right)=$ $s\left(x^{*}, F(t)\right)$ on $[a, b]$ for each $x^{*} \in X^{*}$. Since $\left\{F_{n}\right\}$ is a monotone increasing sequence of Denjoy-Pettis integrable $C W K(X)$-valued mappings on $[a, b]$, $\left\{s\left(x^{*}, F_{n}(\cdot)\right)\right\}$ is a monotone increasing sequence of Denjoy integrable functions on $[a, b]$ for each $x^{*} \in X^{*}$. Let $\lim _{n \rightarrow \infty}(D P) \int_{a}^{b} F_{n}(t) d t=W$, where $W \in C W K(X)$. Then

$$
\begin{aligned}
& \lim _{n \rightarrow \infty} H\left((D P) \int_{a}^{b} F_{n}(t) d t, W\right) \\
= & \lim _{n \rightarrow \infty} \sup _{\left\|x^{*}\right\| \leq 1}\left|s\left(x^{*},(D P) \int_{a}^{b} F_{n}(t) d t\right)-s\left(x^{*}, W\right)\right| \\
= & \lim _{n \rightarrow \infty} \sup _{\left\|x^{*}\right\| \leq 1}\left|\int_{a}^{b} s\left(x^{*}, F_{n}(t)\right) d t-s\left(x^{*}, W\right)\right|=0 .
\end{aligned}
$$

Hence $\lim _{n \rightarrow \infty} \int_{a}^{b} s\left(x^{*}, F_{n}(t)\right) d t=s\left(x^{*}, W\right)$ for each $x^{*} \in B_{X^{*}}$ and so $x^{*} \in$ $X^{*}$. Since $W \in C W K(X), s\left(x^{*}, W\right)$ is finite for each $x^{*} \in X^{*}$. Thus $\lim _{n \rightarrow \infty} \int_{a}^{b} s\left(x^{*}, F_{n}(t)\right) d t$ is finite for each $x^{*} \in X^{*}$. Since $\left\{F_{n}\right\}$ is monotone increasing on $[a, b],\left\{s\left(x^{*}, F_{n}(\cdot)\right)-s\left(x^{*}, F_{1}(\cdot)\right\}\right.$ is a monotone increasing sequence of nonnegative Denjoy integrable functions on $[a, b]$ for each $x^{*} \in X^{*}$. Hence for each subinterval $[c, d]$ of $[a, b]$

$$
\begin{aligned}
& \lim _{n \rightarrow \infty} \int_{c}^{d}\left\{s\left(x^{*}, F_{n}(t)\right)-s\left(x^{*}, F_{1}(t)\right\} d t\right. \\
\leq & \lim _{n \rightarrow \infty} \int_{a}^{b}\left\{s\left(x^{*}, F_{n}(t)\right)-s\left(x^{*}, F_{1}(t)\right\} d t\right. \\
= & \lim _{n \rightarrow \infty} \int_{a}^{b} s\left(x^{*}, F_{n}(t)\right) d t-\int_{a}^{b} s\left(x^{*}, F_{1}(t)\right) d t
\end{aligned}
$$

for each $x^{*} \in X^{*}$. Since $\lim _{n \rightarrow \infty} \int_{a}^{b} s\left(x^{*}, F_{n}(t)\right) d t$ is finite for each $x^{*} \in X^{*}$, for each subinterval $[c, d]$ of $[a, b], \lim _{n \rightarrow \infty} \int_{c}^{d} s\left(x^{*}, F_{n}(t)\right) d t$ is also finite for each $x^{*} \in X^{*}$. By the Monotone Convergence Theorem for the Denjoy integral, for each $x^{*} \in X^{*}, s\left(x^{*}, F(\cdot)\right)$ is Denjoy integrable on $[a, b]$ and for every subinterval $[c, d]$ of $[a, b]$

$$
\lim _{n \rightarrow \infty} \int_{c}^{d} s\left(x^{*}, F_{n}(t)\right) d t=\int_{c}^{d} s\left(x^{*}, F(t)\right) d t .
$$


Using the same method as in the proof of Theorem 3.7, we obtain that $F:[a, b] \rightarrow C W K(X)$ is Denjoy-Pettis integrable on $[a, b]$. Since $F_{n}$ and $F$ are measurable on $[a, b], H\left(F_{n}, F\right)$ is measurable on $[a, b]$ for each $n \in \mathbb{N}$. Since $\left\{F_{n}\right\}$ is monotone increasing on $[a, b]$ and $\lim _{n \rightarrow \infty} s\left(x^{*}, F_{n}(t)\right)=s\left(x^{*}, F(t)\right)$ on $[a, b]$ for each $x^{*} \in X^{*}$,

$$
\begin{aligned}
H\left(F_{n}(t), F(t)\right) & =\sup _{\left\|x^{*}\right\| \leq 1}\left|s\left(x^{*}, F_{n}(t)\right)-s\left(x^{*}, F(t)\right)\right| \\
& \geq \sup _{\left\|x^{*}\right\| \leq 1}\left|s\left(x^{*}, F_{n+1}(t)\right)-s\left(x^{*}, F(t)\right)\right| \\
& =H\left(F_{n+1}(t), F(t)\right)
\end{aligned}
$$

on $[a, b]$ for each $n \in \mathbb{N}$. In particular, $H\left(F_{n}(t), F(t)\right) \leq H\left(F_{1}(t), F(t)\right)$ on $[a, b]$ for each $n \in \mathbb{N}$. Since $H\left(F_{1}, F\right)$ is bounded on $[a, b], H\left(F_{n}, F\right)$ is Lebesgue integrable and so Denjoy integrable on $[a, b]$ for each $n \in \mathbb{N}$. Hence we have

$$
\begin{aligned}
& H\left((D P) \int_{a}^{b} F_{n}(t) d t,(D P) \int_{a}^{b} F(t) d t\right) \\
= & \sup _{\left\|x^{*}\right\| \leq 1}\left|s\left(x^{*},(D P) \int_{a}^{b} F_{n}(t) d t\right)-s\left(x^{*},(D P) \int_{a}^{b} F(t) d t\right)\right| \\
= & \sup _{\left\|x^{*}\right\| \leq 1}\left|\int_{a}^{b} s\left(x^{*}, F_{n}(t)\right) d t-\int_{a}^{b} s\left(x^{*}, F(t)\right) d t\right| \\
\leq & \sup _{\left\|x^{*}\right\| \leq 1} \int_{a}^{b}\left|s\left(x^{*}, F_{n}(t)\right)-s\left(x^{*}, F(t)\right)\right| d t \\
\leq & \int_{a}^{b} \sup _{\left\|x^{*}\right\| \leq 1}\left|s\left(x^{*}, F_{n}(t)\right)-s\left(x^{*}, F(t)\right)\right| d t \\
= & \int_{a}^{b} H\left(F_{n}(t), F(t)\right) d t
\end{aligned}
$$

for each $n \in \mathbb{N}$. By the Monotone Convergence Theorem for the Denjoy integral we have

$\lim _{n \rightarrow \infty} H\left((D P) \int_{a}^{b} F_{n}(t) d t,(D P) \int_{a}^{b} F(t) d t\right) \leq \lim _{n \rightarrow \infty} \int_{a}^{b} H\left(F_{n}(t), F(t)\right) d t=0$.

Thus

$$
\lim _{n \rightarrow \infty} H\left((D P) \int_{a}^{b} F_{n}(t) d t,(D P) \int_{a}^{b} F(t) d t\right)=0 .
$$

\section{References}

[1] R. J. Aumann, Integrals of set-valued functions, J. Math. Anal. Appl. 12 (1965), 1-12

[2] B. Cascales and J. Rodriguez, Birkhoff integral for multi-valued functions, J. Math. Anal. Appl. 297 (2004), 540-560. 
CONVERGENCE THEOREMS FOR SET-VALUED DP-INTEGRABLE MAPPINGS 237

[3] G. Debreu, Integration of Correspondences, Univ. California Press, Berkeley, CA, 1967.

[4] L. Di Piazza and K. Musial, A decomposition theorem for compact-valued Henstock integral, Monatsh. Math. 148 (2006), 119-126.

[5] _ Set-valued Kurzweil-Henstock-Pettis integral, Set-Valued Analysis 13 (2005), $167-179$

[6] K. El Amri and C. Hess, On the Pettis integral of closed valued multifunctions, SetValued Analysis 8 (2000), 329-360.

[7] J. L. Gamez and J. Mendoza, On Denjoy-Dunford and Denjoy-Pettis integrals, Studia Math. 130 (1998), 115-133.

[8] R. A. Gordon, The Denjoy extension of the Bochner, Pettis and Dunford integrals, Studia Math. 92 (1989), 73-91.

[9] _ The Integrals of Lebesgue, Denjoy, Perron and Henstock, Grad. Stud. Math. 4, Amer. Math. Soc. Providence, R.I., 1994.

[10] N. Papageoriou, On the theory of Banach space valued multifunctions, J. Multivariate Anal. 17 (1985), 185-206.

[11] S. Saks, Theory of the Integral, Dover, New York, 1964.

[12] J. Wu and C. Wu, The w-derivatives of fuzzy mappings in Banach spaces, Fuzzy Sets and Systems 119 (2001), 375-381.

[13] W. Zhang, Z. Wang, and Y. Gao, Set-Valued Stochastic Process, Academic Press, Beijing, 1996.

Department of Mathematics

KANGWON National University

Chuncheon 200-701, Korea

E-mail address: ckpark@kangwon.ac.kr 